\title{
Singular Value Decomposition and Ligand Binding Analysis
}

\author{
André Luiz Galo and Márcio Francisco Colombo \\ Department of Physics, IBILCE, São Paulo State University (UNESP), Rua Cristóvão Colombo 2265, Jardim Nazareth, \\ 15054-000 São José do Rio Preto, SP, Brazil \\ Correspondence should be addressed to André Luiz Galo; galo@ibilce.unesp.br
}

Received 20 May 2013; Revised 26 August 2013; Accepted 28 August 2013

Academic Editor: Adam F. Lee

Copyright ( 2013 A. L. Galo and M. F. Colombo. This is an open access article distributed under the Creative Commons Attribution License, which permits unrestricted use, distribution, and reproduction in any medium, provided the original work is properly cited.

\begin{abstract}
Singular values decomposition (SVD) is one of the most important computations in linear algebra because of its vast application for data analysis. It is particularly useful for resolving problems involving least-squares minimization, the determination of matrix rank, and the solution of certain problems involving Euclidean norms. Such problems arise in the spectral analysis of ligand binding to macromolecule. Here, we present a spectral data analysis method using SVD (SVD analysis) and nonlinear fitting to determine the binding characteristics of intercalating drugs to DNA. This methodology reduces noise and identifies distinct spectral species similar to traditional principal component analysis as well as fitting nonlinear binding parameters. We applied SVD analysis to investigate the interaction of actinomycin D and daunomycin with native DNA. This methodology does not require prior knowledge of ligand molar extinction coefficients (free and bound), which potentially limits binding analysis. Data are acquired simply by reconstructing the experimental data and by adjusting the product of deconvoluted matrices and the matrix of model coefficients determined by the Scatchard and McGee and von Hippel equation.
\end{abstract}

\section{Introduction}

The determination of association constants of binding between biological macromolecules and their ligands is of fundamental importance for the characterization of the energetics of biochemical reactions. Without this knowledge, it is not possible, for example, to determine biological regulatory mechanisms to design stereochemical models to comprehensively describe the molecular interactions that determine specificity, reactivity, and allosteric regulation typical of biochemical reactions.

Investigators use a large range of experimental methods based on physical differences between bound and free ligands to determine binding constants. Among these, UV-Vis spectroscopic methods are widely used [1-4]. Two factors favor these techniques as follows. First, ligands and biological macromolecules frequently contain chromophores, which absorb light in the UV-Vis region and can be used as reporters of ligand binding. Second, the techniques are relatively simple and inexpensive and generally do not require large amounts of material. However, limitations in determining association constant using spectroscopy arise when the spectral characteristics of bound species are unknown or when affinity is relatively high. In the latter case, it may be difficult to reliably determine the concentration of unbound ligands.

Another possible limitation is the spectrophotometric detection limit, which can be reduced by increasing the optical path length using, for example, liquid core waveguide (LCW) [5]. LCW was proposed as an effective solution to detect molecules at very low optical densities $[5,6]$. However, the increasing optical path length employed by the LCW strategy does not minimize errors associated with hyperbolic equation linearization frequently used in a classical analysis using double-reciprocal or Scatchard plots. These errors are more apparent at low binding ratios and require nonlinear fitting $[1,7]$.

Knowledge of the concentrations of free and bound ligands is required for the methods described above and typically required measurements at a single wavelength, 
where the bound $\left(C_{B}\right)$ and free $\left(C_{F}\right)$ concentrations are given by the Beer-Lambert law as follows [1]:

$$
C_{B}=\frac{C_{T} \varepsilon_{F}-A / \ell}{\varepsilon_{F}-\varepsilon_{B}},
$$

where $C_{F}=C_{T}-C_{B}$ and $C_{T}$ represents total ligand concentration; $\varepsilon_{B}$ and $\varepsilon_{F}$ are the bound and free molar extinction coefficients, respectively; $A$ is the absorbance; and $\ell$ is the optical path length. However, prior knowledge of bound and free molar extinction coefficients is required.

Without the exact knowledge of the spectra of bound and free ligands, the strong dependence of the model with ligand distribution, experimental data obtained near the detection limit, or high noise or scattering (for a long path length) may impair the ability to determine binding parameters with reproductivity and confidence, particularly when the ligand/macromolecule ratio $(r)$ is low. The high-affinity binding of actinomycin D (ActD) to DNA represents such an example [8-11]. In these studies, the divergence between the results reported by different research groups generates a very low $r$ value, whereas larger $r$ values indicate concordance.

The problems described above can be resolved using a nonlinear fitting model linked to singular value decomposition (SVD) analysis of spectral data. SVD analysis is commonly used in UV-Vis spectroscopy and provides higher accuracy for solving problems involving least-squares minimization and other problems involving Euclidian norms [12]. Several studies employ SVD for spectral analysis of ligand binding in chemical and biochemical reactions [1316]. However, in most of cases, SVD spectral analysis is used only to determine the number of distinct spectral species associated with the course of the reaction or to minimize spectral noise, though, the binding model can be established using SVD spectral data analysis to determine binding parameters.

In the present study, we systematically describe SVD binding analysis of ligand-biological macromolecule interactions (drug-DNA) and compare the data with those acquired using standard Scatchard plots. We show that a generic binding model (e.g., Scatchard or McGee and von Hippel) can be used without prior knowledge of the ligand's bound and free molar extinction coefficients.

\section{Materials and Methods}

Let us first consider the spectra of a sample comprising $n$ species. The observed absorption (UV-Vis-Nir) of a sample, at a given wavelength $\lambda$ with intrinsic coefficient of extinction $\varepsilon_{i}(\lambda)$, may be quantitatively expressed within the validity of the Beer-Lambert law by the following equation:

$$
A(\lambda)=\frac{\operatorname{Abs}(\lambda)}{\ell}=C_{1} \varepsilon_{1}(\lambda)+C_{2} \varepsilon_{2}(\lambda)+\cdots+C_{n} \varepsilon_{n}(\lambda),
$$

where $C_{i}$ is the concentration of the $i$ th ligand molecule and $\ell$ is the optical path length. When $\varepsilon_{i}(\lambda)$ is the vector of the extinction coefficient measured at $m$ wavelengths, $A(\lambda)$ represents the spectrum of the absorbance of the sample and can be written in matrix form as follows:

$$
\mathbf{A}_{\mathbf{s}}=\mathbf{E C}^{\mathbf{T}}=\left(\begin{array}{cccc}
\varepsilon_{1}^{\lambda_{1}} & \varepsilon_{2}^{\lambda_{1}} & \cdots & \varepsilon_{n}^{\lambda_{1}} \\
\varepsilon_{1}^{\lambda_{2}} & \varepsilon_{2}^{\lambda_{2}} & \cdots & \varepsilon_{n}^{\lambda_{2}} \\
\vdots & \vdots & \ddots & \vdots \\
\varepsilon_{1}^{\lambda_{m}} & \varepsilon_{2}^{\lambda_{m}} & \cdots & \varepsilon_{n}^{\lambda_{m}}
\end{array}\right) \cdot\left(\begin{array}{c}
C_{1} \\
C_{2} \\
\vdots \\
C_{n}
\end{array}\right),
$$

where $\mathbf{E}$ is the $m$ (wavelength) $\times n$ (species) extinction coefficient matrix column and $\mathbf{C}$ is a matrix row of the concentration of $n$ species.

In a classical spectrophotometric titration experiment, the absorption spectra are measured at each titration step. These data can be organized in a matrix $\mathbf{A}$ :

$$
\mathbf{A}=\left(\begin{array}{ccc}
A\left(\lambda_{1}\right)^{1} & \cdots & A\left(\lambda_{1}\right)^{n} \\
\vdots & \ddots & \vdots \\
A\left(\lambda_{m}\right)^{1} & \cdots & A\left(\lambda_{m}\right)^{n}
\end{array}\right),
$$

where each column of the matrix $\mathbf{A}$ corresponds to the spectrum of each titration. Assuming that the interaction of ligand macromolecule is effectively characterized by only two spectral species (bound and free), the absorbance per wavelength in each experimental condition is given as follows:

$$
A(\lambda)=f_{F} \varepsilon_{F}(\lambda)+f_{B} \varepsilon_{B}(\lambda)+\sum_{n} O^{n}(\lambda),
$$

where the ligand-free fraction $f_{F}$ and ligand-bound fraction $f_{B}$ are determinant factors for global spectral measurement and are given by the binding model. The $O^{n}(\lambda)$ terms are lower orders of any negligible data (noise).

In the example of (2), the experimental data matrix $\mathbf{A}$ can be decomposed into two factors as follows: $\mathbf{A}=\mathbf{D F} \mathbf{F}^{\mathrm{T}}$, where the $\mathbf{D}$ matrix represents the molar extinction coefficients, and the $\mathbf{F}$ matrix represents the free and bound ligand fractions. We can also decompose the A matrix into singular values,

$$
\mathbf{A}=\mathbf{D F}^{\mathrm{T}}=\mathbf{U S V}^{\mathrm{T}},
$$

where $\mathbf{U}^{\mathrm{T}} \mathbf{U}=\mathbf{V}^{\mathrm{T}} \mathbf{V}=\mathbf{I}$. $\mathbf{U}$ and $\mathbf{V}$ are orthogonal (unitary), $\mathbf{S}$ is diagonal, and the mean diagonal values of $\mathbf{S}$ matrix are called singular values of $\mathbf{S}$.

In particular, the most important aspect of this decomposition is that the matrix $\mathbf{U}$ (eigenvectors of $\mathbf{A A ^ { T }}{ }^{\mathrm{T}}$ ) contains so-called basis spectra, and the matrix $\mathbf{V}$ (eigenvectors of $\left.\mathbf{A}^{\mathrm{T}} \mathbf{A}\right)$ contains the amplitude vectors. The singular values (eigenvalues of $\mathbf{A}^{\mathrm{T}} \mathbf{A}$ e $\mathbf{A A}^{\mathrm{T}}$ ) determine the weight associated with each basis spectra for the global spectra $[15,16]$. Defined as the rank $\mathbf{S}$, that is, the minimal number of singular values for data representation, the data matrix can be rewritten as $\mathbf{U S}_{1} \mathbf{V}^{\mathrm{T}}$, where $\mathbf{S}_{1}$ represents the rank matrix containing only a significant singular value matrix without spurious information.

The matrix $\mathbf{D}$ can be rewritten as follows:

$$
\mathbf{D}=\mathbf{U S V}^{\mathrm{T}} \mathbf{F}^{\mathrm{T}+}=\mathbf{U S H},
$$

where $\mathbf{F}^{\mathrm{T}+}$ is the pseudoinverse of $\mathbf{F}^{\mathrm{T}}$ and $\mathbf{H}=\mathbf{V}^{\mathrm{T}} \mathbf{F}^{\mathrm{T}+}$. 
Consequently

$$
\mathbf{A}=\mathbf{D F}^{\mathrm{T}}=\mathrm{USHF}^{\mathrm{T}}=\mathbf{U S V}^{\mathrm{T}}
$$

or

$$
\mathbf{V}^{\mathrm{T}}=\mathrm{HF}^{\mathrm{T}}
$$

Therefore, $\mathbf{H}$ can be defined by matrices $\mathbf{V}$ and $\mathbf{F}$, and these, in turn, are defined by the SVD of the experimental data (matrix A). However, $\mathbf{F}$ can be defined from the bound and free fractions given by the equilibrium model. For example, taking into consideration the Scatchard model $\left(C_{B} /[\mathrm{DNA}] C_{F}=\right.$ $\left.n K /\left(1+K C_{F}\right)\right)$, we derive the equation as follows:

$$
-K C_{F}^{2}+C_{F}\left(K C_{T}-[M] n K-1\right)+C_{T}=0,
$$

where the equation solution (i.e., zero function) determines the free-species concentration $\left(C_{F}\right)$ as a function of the binding constant $K$, average number of binding sites $n$, and DNA concentration (DNA) parameters in which the total concentration is given by the sum of free and bound concentrations $\left(C_{T}=C_{F}+C_{B}\right)$.

Thus, we can solve the binding parameters by setting values of $n$ and $K$ that minimize the norm

$$
\left\|\mathbf{V}^{\mathrm{T}}-\mathbf{H F}^{\mathrm{T}}\right\|
$$

where $\mathbf{V}$ and $\mathbf{H}$ represent experimental SVD data and $\mathbf{F}$ is fitted by the equilibrium model. This routine was implemented in the MATLAB (The MathWorks, Inc.) program and can be accessed in Supplementary Materials available online at http://dx.doi.org/10.1155/2013/372596.

2.1. Materials and Data Acquisition. The naturally occurring antibiotics ActD (EMD Millipore) and daunomycin (Daun, Sigma-Aldrich) were used without further purification. Phenol-extracted calf thymus DNA (ct-DNA, GE Life Sciences) was dissolved in $10 \mathrm{mM}$ phosphate buffer, $\mathrm{pH} 7.5$, containing $150 \mathrm{mM} \mathrm{NaCl}$ and $1 \mathrm{mM}$ EDTA. The DNA solutions were passed through $0.25 \mu \mathrm{m}$ Millipore filters before the binding experiments. Drug concentrations were determined spectrophotometrically using molar extinction coefficients $\varepsilon_{F}=24800 \mathrm{M}^{-1} \mathrm{~cm}^{-1}$ at $440 \mathrm{~nm}$ for ActD $[4,14]$ and $\varepsilon_{F}=$ $7000 \mathrm{M}^{-1} \mathrm{~cm}^{-1}$ at $480 \mathrm{~nm}$ for Daun [15]. The ct-DNA concentrations were then determined using the molar extinction coefficient at $260 \mathrm{~nm}$ of $\varepsilon=12800 \mathrm{M}^{-1} \mathrm{~cm}^{-1} \mathrm{bp}^{-1}[4,14]$. Binding isotherms were measured by spectrophotometric titration using a Cary-3E spectrophotometer (Varian, Inc.) and $50 \mathrm{~mm}$ path-length quartz cuvettes at room temperature $\left(25^{\circ} \mathrm{C}\right)$. Small aliquots of the drug were added to a DNA solution in a cuvette (drug: DNA ratio, $10^{-2}-10^{-1}$ ), and drug absorbance spectra were measured at 30 to $60 \mathrm{~min}$ after adding the drug.

\section{Results and Discussion}

Our initial goal was to assess the potential of SVD binding analysis for analyzing the binding of drugs to DNA. We

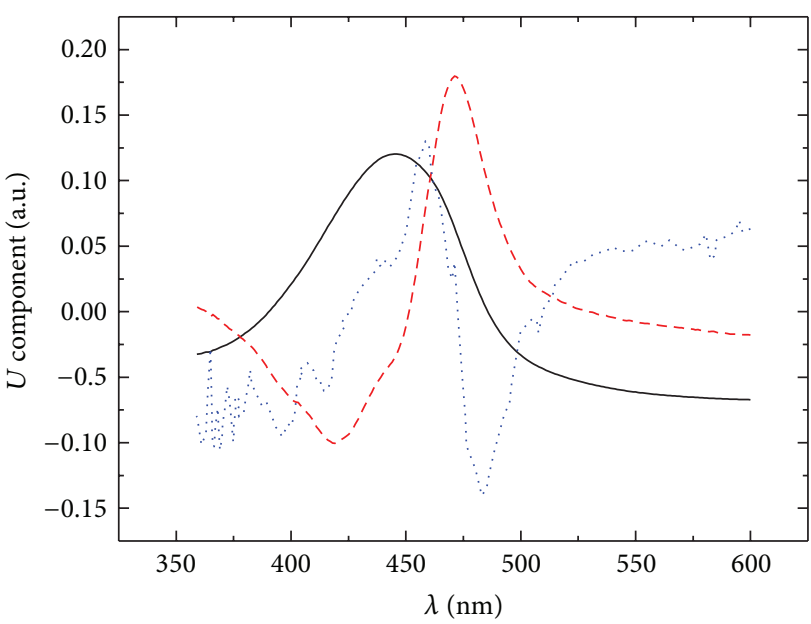

FIGURE 1: The first three $U$ component spectra in sequence: solid, dashed, and dotted lines for the SVD of binding of Act-D-DNA spectrophotometric titration data.

TABLE 1: The first five singular values (S matrix principal components) for ActD-DNA spectrophotometric titration data.

\begin{tabular}{lc}
\hline Singular values & Percentage $(\%)$ \\
\hline 9.64607 & 98.0741 \\
1.35081 & 1.9233 \\
0.04669 & 0.0023 \\
0.01355 & 0.0002 \\
0.00678 & 0.0000 \\
\hline
\end{tabular}

therefore conducted spectrophotometric titrations of ActD and Daun binding to ct-DNA. We used standard buffer conditions and a typical range of drug: DNA ratios according to accepted published values $[1,8]$.

The first step for applying SVD binding analysis was to compute the singular value of the experimental matrix $\mathbf{A}$ and define the rank of matrix $\mathbf{S}$. The first three $U$ component spectra are shown in Figure 1, and the first five singular values (diagonal components of the matrix $\mathbf{S}$ ) and their weights are shown in each column of Table 1.

The spectral data can be represented for only the two first singular values, describing $99 \%$ and $98 \%$ of their observed behavior. According to the data presented in Table 1 and the correlation of $V$ components (Figures 2 and 3), we conclude that only the first and second singular values are relevant to reconstruct the experimental matrix $\mathbf{A}$ and that the others correlated with noise. We note that these two equal ranks indicate that they represent only two distinct spectra of ActD, one of the free drug and the other of drug intercalated in DNA. The first and second columns of matrix $\mathbf{U}$ represent compositors of the original spectra of free and bound drug. The values of $V$ and $S$ are weighted according to only first and second elements of diagonal matrix $\mathbf{S}$ for combining all the information required to describe the experimental data, while the remaining terms can be considered as noise.

Therefore, we display details only as the first two SVD components. For Figure 2 shows the $V$-plot components of 


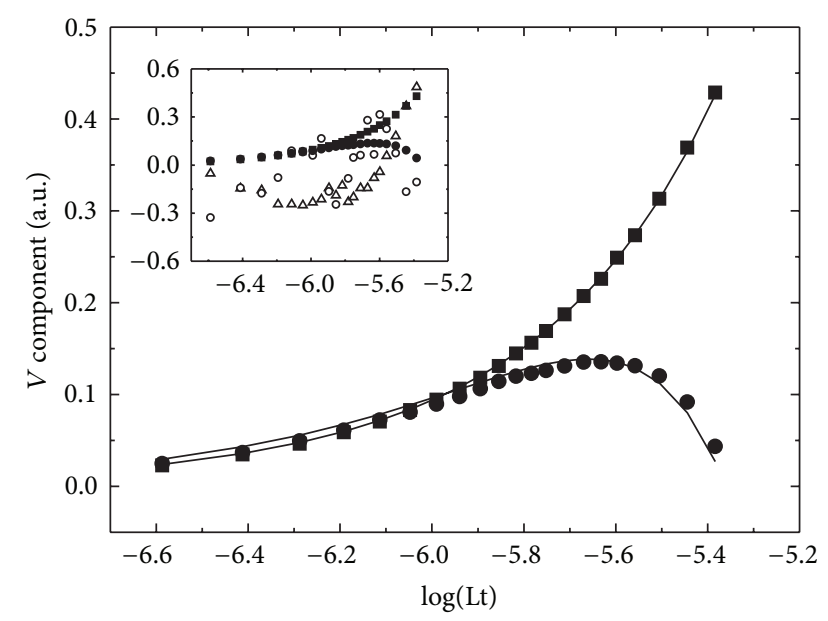

Figure 2: $V$ component amplitude as a function of the total ActD concentration. The symbols represent values, of the SVD matrix for the ActD-DNA titration. The squares represent the first $V$ component values and the circles represent the amplitudes of the second component. The first four $V$ component amplitudes are shown in the inset, and the results generated from $\mathbf{F}$ matrix were adjusted to the experimental data according to SVD analysis routine (solid lines).

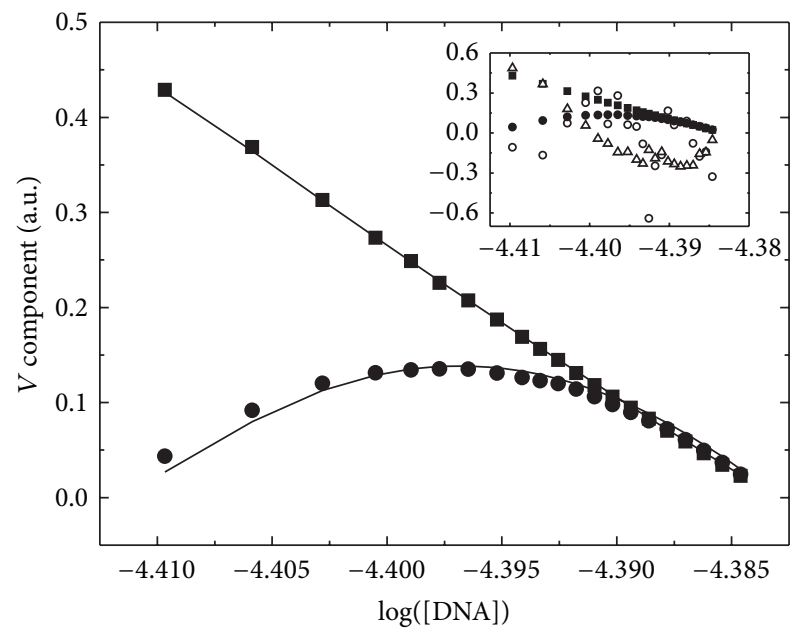

Figure 3: $V$ component amplitude as a function of the total DNA concentration. Data were generated from the SVD matrix of the ActD-DNA titration data. The squares show the first $V$ component values, and circles represent the second-component amplitudes. The first four $V$ component amplitudes are shown in the inset, and the results generated from the $\mathbf{F}$ matrix were adjusted to the experimental data using our SVD analysis routine and are shown by the solid lines.

the experimental data matrix $\mathbf{A}$ as a function of the logarithm of total ligand concentration (symbols). In this figure, the solid lines are given by the $\mathbf{H F}^{\mathrm{T}}$ matrix, and the $\mathbf{F}$ matrix is defined by the fitted equilibrium model (one site Scatchard). Figure 3 shows the $V$ components (symbols) and the fitted model (solid lines) as a function of the DNA concentration.

Usually, the binding curve can be determined using classical procedures such as the Scatchard plot and computed using

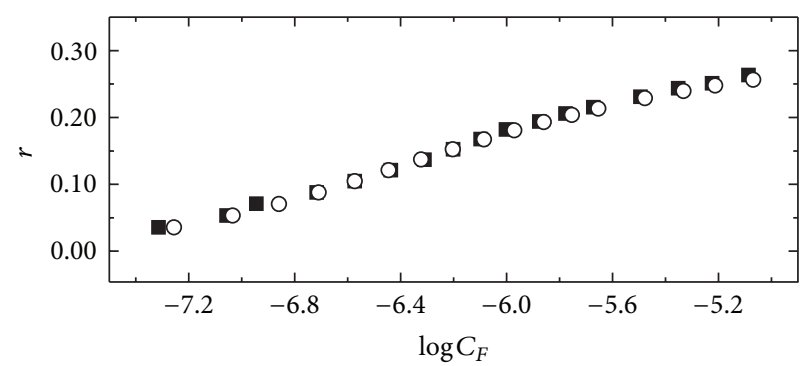

(a)

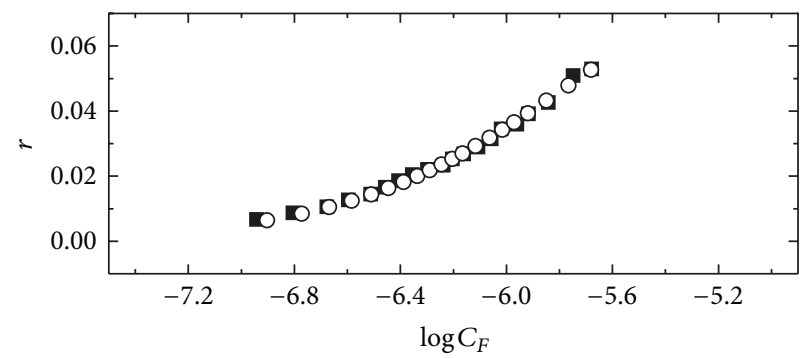

(b)

FIGURE 4: The rate number $r$ (ratio of bound ligand concentration to DNA concentration) as a function of the free ligand concentration for ActD-DNA titration. The squares and circles represent the classical and SVD model fitting procedures, respectively.

a nonlinear model-fitting algorithm (standard procedure); however, using one point in the absorbance spectra (1) can introduce errors. In SVD binding analysis, the fitted $\mathbf{F}$ matrix gives the free and bound ligand concentrations, with the advantage that model fitting uses the global spectra (all visible wavelengths) and associates each linearly independent component with one spectral species (free and bound spectra), thus minimizing spectral noise.

Figure 4 shows the binding curve $\left(r \times \log \left[C_{F}\right]\right)$ determined by two methods, a standard procedure and SVD binding analysis for ActD (top) and Daun (bottom) to DNA binding.

In the SVD binding analysis, the extinction coefficients of all distinct species are given by the $\mathbf{D}$ matrix defined by (6). Figure 5 shows the spectra of the free and bound molar extinction coefficients determined by SVD binding analysis for ActD binding to DNA and the free DNA-ActD sample spectra. We note that for the SVD binding analysis, previous knowledge of the free and bound ligand spectra is not required, because these are given by model fitting. This figure shows that the free spectra of ActD generated by fitting correlate highly with the free ActD spectra. Figure 6 shows similar results for Daun binding to DNA.

The absorbance spectra (matrix A) can be reproduced from numerical fitting data; that is, $\mathbf{A}_{\mathrm{Fit}}=\mathbf{D F}^{\mathrm{T}}$. The recalculated spectra $\left(\mathbf{A}_{\mathrm{Fit}}\right)$; and the residual spectra $\left(\mathbf{A}_{\mathrm{Fit}}-\right.$ $\mathbf{A}_{\text {Exp }}$ ), and differences between recalculated and measured spectra are shown in Figure 7. Most of residual spectra are below $4.0 \times 10^{-3}$, and the rms fluctuation of all residual spectra present equals $1.5 \times 10^{-3}$, the same magnitude as the experimental noise. 


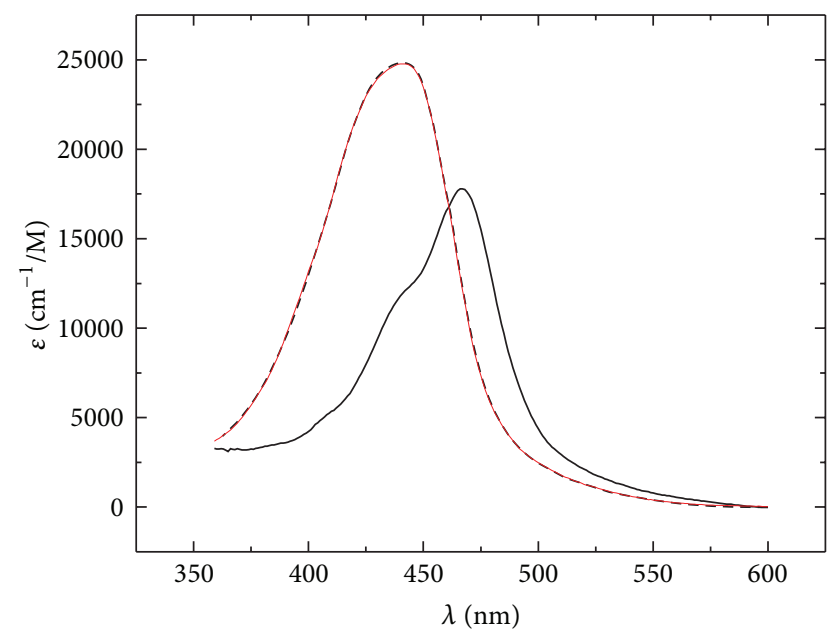

FIGURE 5: Free (dashed line) and bound (solid line) molar extinction coefficient spectra determined by the SVD fitting model for ActD compared with the free DNA-ActD sample extinction coefficient spectra (thin solid line).

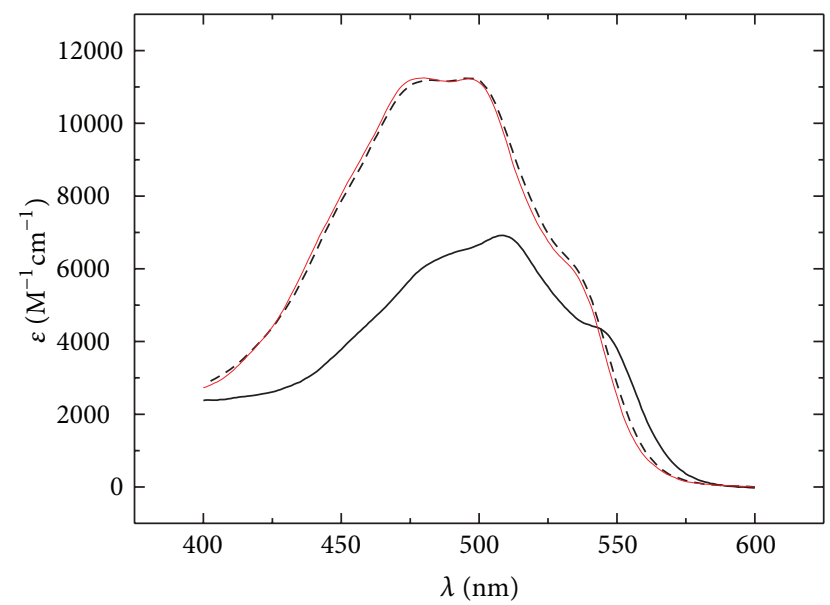

FIGURE 6: Free (dashed line) and bound (solid line) molar extinction coefficient spectra determined by the SVD fitting model for Daun compared with free DNA-Daun sample extinction coefficient spectra (soft solid line).

The data generated by the classical and SVD methods shown in Figure 5 are nearly indistinguishable. Although Scatchard plot-fitting is more susceptible to errors at low values of $r$, this plot highlights the differences between the standard method and SVD binding analysis. Figure 8 shows the Scatchard plot obtained by standard methodology $[1,8]$ (circles). A linear fitting (one identical and independent site model) gives the association constant $K=7.0 \pm 0.5 \times 10^{5} \mathrm{M}^{-1}$ and the number of base pairs occupied $n=12 \pm 1 \mathrm{bp} /$ (bound drug molecule). The nonlinear fitting results from SVD binding analysis are shown as a Scatchard plot in Figure 8 (squares). The SVD binding analysis gives the association constant $K=5.57 \pm 0.07 \times 10^{5} \mathrm{M}^{-1}$ and the number of base pairs occupied $n=10 \mathrm{bp} /$ (bound drug molecule). The Scatchard plot reveals a slight discrepancy at low $r$ that is

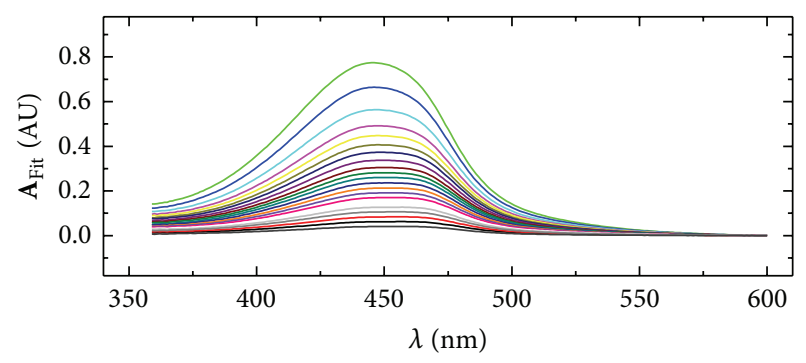

(a)

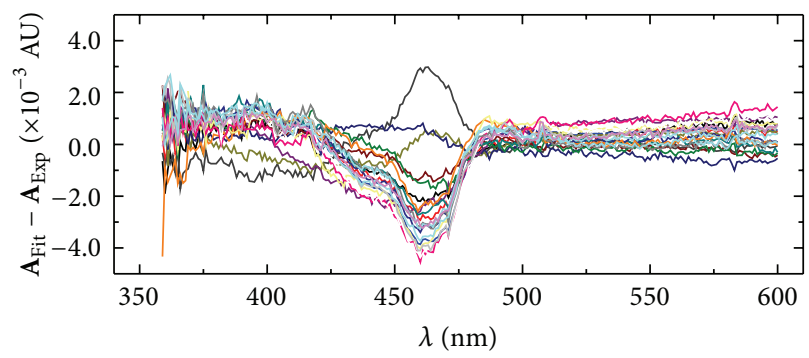

(b)

Figure 7: (a) Absorbance spectra recalculated using $\mathbf{A}_{\mathrm{Fit}}=\mathbf{D F} \mathbf{F}^{\mathrm{T}}$, where $\mathbf{F}$ is given by the fitted model. (b) The residual absorbance spectra $\left(\mathbf{A}_{\mathrm{Fit}}-\mathbf{A}_{\mathrm{Exp}}\right)$ of the difference between the recalculated and experimental spectra.

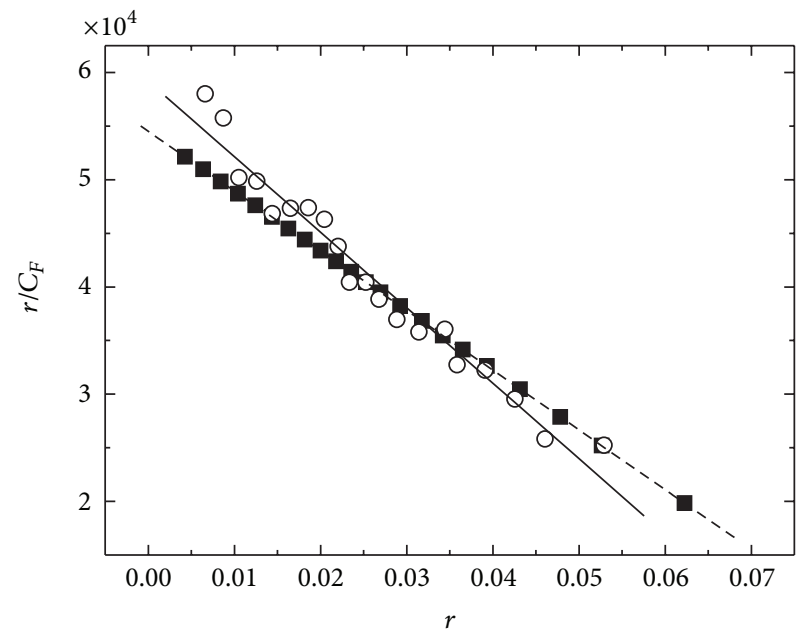

FIgURE 8: Scatchard plot of ActD-DNA binding. The squares and circles represent the SVD binding and standard procedures, respectively. The one-site model fitting using the Scatchard plot gives $K=7.0 \pm 0.5 \times 10^{5} \mathrm{M}^{-1}$ and $n=12 \pm 1 \mathrm{bp} /$ (bound drug), while the SVD binding analysis gives $K=5.57 \pm 0.07 \mathrm{M}^{-1} \times 10^{5} \mathrm{M}^{-1}$ and $n=10 \mathrm{bp} /$ (bound drug).

responsible for the increase in slope. This can be attributed to different methodologies, because the standard method uses only one spectral point. In contrast, the SVD binding analysis uses the total spectral data and is, in principle, more precise and less sensitive to noise.

A similar analysis for Daun binding to DNA is shown in Figure 9. The noncooperative McGee and von Hippel model 


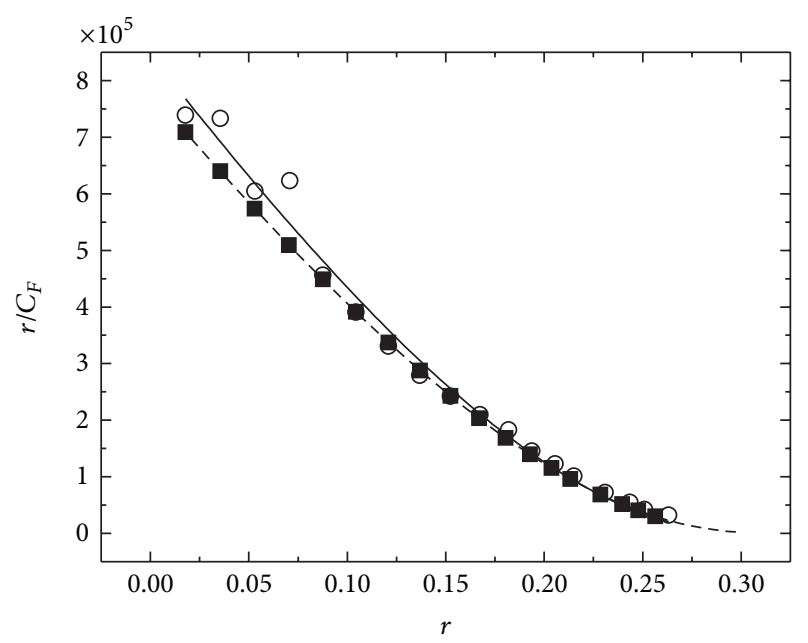

Figure 9: Scatchard plot of Daun-DNA binding. The squares and circles show SVD-based fitting and a traditional Scatchard plot, respectively. The standard noncooperative McGee and von Hippel model fitting parameters are $K=8.5 \pm 0.2 \times 10^{5} \mathrm{M}^{-1}$ and $n=3.14 \pm$ $0.06 \mathrm{bp} /$ (bound drug molecule) while the SVD-based parameters for fitting are $K=7.79 \pm 0.05 \times 10^{5} \mathrm{M}^{-1}$ and $n=3.0 \mathrm{bp} /$ (bound drug molecule).

was fitted to the data obtained by the standard method, and the SVD binding analysis results are plotted on the same graph. This standard analysis gives the binding parameters $K=8.5 \pm 0.2 \times 10^{5} \mathrm{M}^{-1}$ and $n=3.14 \pm 0.06 \mathrm{bp} /$ (bound drug molecule), and the SVD binding analysis gives $K=7.79 \pm$ $0.05 \times 10^{5} \mathrm{M}^{-1}$ and $n=3.0 \mathrm{bp} /$ (bound drug molecule). In both cases, it is apparent that the SVD binding analysis significantly reduced the error in determining binding parameters.

3.1. Validation Methodology. The SVD binding analysis methodology described above gave a free molar extinction consistent with values measured directly (Figure 5). Therefore, determining the extinction coefficient of the DNAbound molecules was required. Without prior knowledge of a drug's absorbance spectrum, extinction coefficients of bound drugs can be obtained by extrapolation of drug absorbance in the presence of a large excess of DNA binding sites. The literature values of the extinctions coefficients $\left(\varepsilon_{B}\right)$ of bound ActD [1, 17] and Daun [18] are $=11900 \mathrm{M}^{-1} \mathrm{~cm}^{-1}$ in $440 \mathrm{~nm}$ and $7000 \mathrm{M}^{-1} \mathrm{~cm}^{-1}$ in $480 \mathrm{~nm}$, respectively. These values agree with our results computed by SVD analysis of $11794 \mathrm{M}^{-1} \mathrm{~cm}^{-1}$ and $6142 \mathrm{M}^{-1} \mathrm{~cm}^{-1}$, respectively.

These results indicate a good fit of the data. Therefore, to confirm these results, extinction coefficient spectra for ActD were determined directly as follows. We placed the ActD samples into two cuvettes in a double-beam spectrophotometer and titrated the samples with DNA (sample cuvette) and reference sample (reference cuvette) in an equal volume of buffer. The measured spectra are calculated using the equation $A=\left(\varepsilon_{B}-\varepsilon_{F}\right) \cdot C_{B}$. The difference in molar extinction

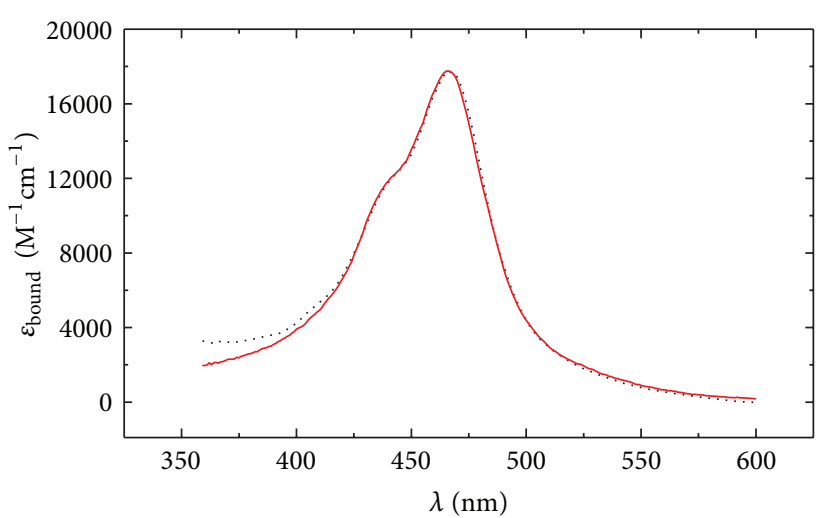

(a)

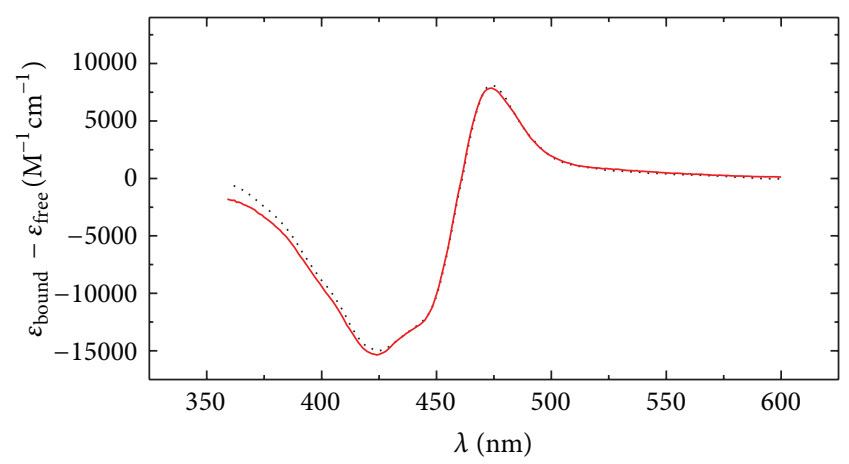

(b)

FIgURE 10: The $\left(\varepsilon_{B}-\varepsilon_{F}\right)$ and $\varepsilon_{B}$ spectra determined using SVDbased binded analysis compared with directly measured coefficients. The solid lines represent the measured data, and the dash lines represent the results for extinctions coefficients determined by SVD and model fitting.

spectra [difference spectra $\left(\varepsilon_{B}-\varepsilon_{F}\right)$ ] is determined by a leastsquares minimization adjustment of $A(\lambda)^{i}=\left[\varepsilon_{b}(\lambda)-\right.$ $\left.\varepsilon_{f}(\lambda)\right] \cdot C_{B}^{i}$, where the coefficient $i$ represents the $i$ th titration and $\lambda$ represents the wavelength. Once the difference spectra are determined, the bound spectra $\left(\varepsilon_{B}\right)$ are given by $\varepsilon_{B}=$ $\left(\varepsilon_{B}-\varepsilon_{F}\right)+\varepsilon_{F}$. Figure 10 shows the difference spectra $\left(\varepsilon_{B}-\right.$ $\varepsilon_{F}$ ) (a) and extinction coefficients spectra $\left(\varepsilon_{B}\right)$ (b) for ActD binding to DNA, calculated using matrix $\mathbf{D}$ according to SVD binding analysis formalism (dashed lines) compared with the experimental procedure described above (solid lines). This result, taken together with the representative described above, validates this technical analysis, and the results presented in Figures 8 and 9 demonstrate the actual potential of SVD binding analysis.

\section{Conclusions}

We show here that SVD binding analysis is an effective analytical tool that produces very good results compared with standard methods and offers the significant advantage of the ability to determine binding constants without prior knowledge of extinctions coefficients. Another major advantage of SVD compared with standard methods that employ 
only a single wavelength to determine drug concentrations is that it uses global spectra that generate more reliable data. Thus, the method presented here determines absorbance over a wide range of wavelengths. Moreover, the binding model to determine free and bound concentrations of ligand reduces noise by fitting only relevant SVD parameters in a simple and direct form. We believe that the data convincingly demonstrate the precision of SVD and its potential application to analysis of ligand binding. Moreover, the molar extinction coefficients determined by SVD binding analysis correlate closely with validated literature values. We conclude therefore that the analytical method developed here is very reliable and may provide an acceptable alternative for studying highaffinity binding systems or may be used when the molar extinction coefficient of the bound ligand is unknown.

\section{Acknowledgments}

The authors thank the Brazilian agencies Capes and CNPq for financial support.

\section{References}

[1] W. Muller and D. M. Crothers, "Studies of the binding of actinomycin and related compounds to DNA," Journal of Molecular Biology, vol. 35, no. 2, pp. 251-290, 1968.

[2] T. Jankins, Drug-DNA Interaction Protocols, Fox K. R., Ed, Humana Press, Totowa, NJ, USA, 1997.

[3] A. L. Galo, J. Rugiero Neto, D. P. Brognaro, R. C. Caetano, F. P. Souza, and M. F. Colombo, "The influence of solutes on the enthalpy/entropy change of the actinomycin d binding to DNA: hydration, energy compensation and long-range deformation on DNA," Journal of Physical Chemistry B, vol. 115, no. 28, pp. 8883-8890, 2011

[4] P. O. Vardevanyan, A. P. Antonyan, M. A. Parsadanyan, M. A. Shahinyan, L. A. Hambardzumyan, and M. A. Torosyan, "The influence of GC/AT composition on intercalating and semiintercalating binding of ethidium bromide to DNA," Journal of the Brazilian Chemical Society, vol. 23, no. 11, pp. 2016-2020, 2012.

[5] R. H. Byrne and E. Kaltenbacher, "Use of liquid core waveguides for long pathlength absorbance spectroscopy: principles and practice," Limnology and Oceanography, vol. 46, no. 3, pp. 740742, 2001.

[6] A. L. Galo and M. Colombo, "Espectrofotometria de longo caminho óptico em espectrofotômetro de duplo-feixe convencional: uma alternativa simples para investigações de amostras com densidade óptica muito baixa," Química Nova, vol. 32, no. 2, p. 488, 2009.

[7] J. G. Norby, P. Ottolenghi, and J. Jensen, "Scatchard plot: common misinterpretation of binding experiments," Analytical Biochemistry, vol. 102, no. 2, pp. 318-320, 1980.

[8] J. Ruggiero Neto and M. F. Colombo, "Water regulation of actinomycin-D binding to DNA: the interplay among drug affinity, DNA long-range conformation, and hydration," Biopolymers, vol. 53, no. 1, pp. 46-59, 2000.

[9] J. Ruggiero Neto, F. Pereira de Souza, and M. F. Colombo, "Hydration effects on DNA double helix stability modulates ligand binding to natural DNA in response to changes in water activity," Cell and Molecular Biology, vol. 47, p. 801, 2001.
[10] J. B. Chaires, "Drug-DNA interactions," Current Opinion in Structural Biology, vol. 8, no. 3, pp. 314-320, 1998.

[11] X. Qu and J. B. Chaires, "Hydration changes for DNA intercalation reactions," Journal of the American Chemical Society, vol. 123, no. 1, pp. 1-7, 2001.

[12] G. H. Golub and C. F. Van Loan, Matrix Computations, Johns Hopkins University Press, Baltimore, Md, USA, 3th edition, 1996.

[13] E. R. Henry and J. Hofrichter, "Singular value decomposition: application to analysis of experimental data," Methods in Enzymology, vol. 210, pp. 129-192, 1992.

[14] E. R. Henry, "The use of matrix methods in the modeling of spectroscopic data sets," Biophysical Journal, vol. 72, no. 2, pp. 652-673, 1997.

[15] K. D. Vandergriff and R. I. Shrager, "[22] Hemoglobin-oxygen equilibrium binding: rapid-scanning spectrophotometry and singular value decomposition," Methods in Enzymology, vol. 232, pp. 460-185, 1994.

[16] M. E. Wall, A. Rechtsteiner, and L. M. Rocha, "Singular value decomposition and principal component analysis," in $A$ Practical Approach to Microarray Data Analysis, D. P. Berrar, W. Dubitzky, and M. Granzow, Eds., pp. 91-109, Kluwer, Norwell, Mass, USA, 2003.

[17] F. Quadrifoglio and V. Crescenzi, "On the binding of actinomycin and of daunomycin to DNA. a calorimetric and spectroscopic investigation," Biophysical Chemistry, vol. 2, no. 1, pp. 64-69, 1974.

[18] J. B. Chaires, N. Dattagupta, and D. M. Crothers, "Studies on interaction of anthracycline antibiotics and deoxyribonucleic acid: equilibrium binding studies on interaction of daunomycin with deoxyribonucleic acid," Biochemistry, vol. 21, no. 17, pp. 3933-3940, 1982. 

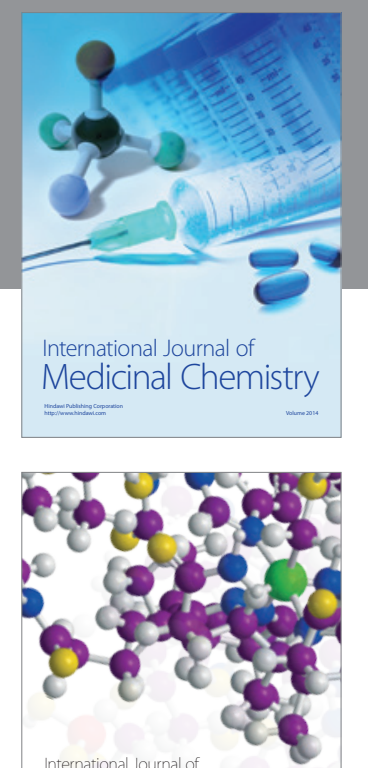

\section{Carbohydrate} Chemistry

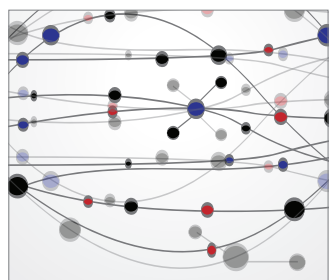

The Scientific World Journal
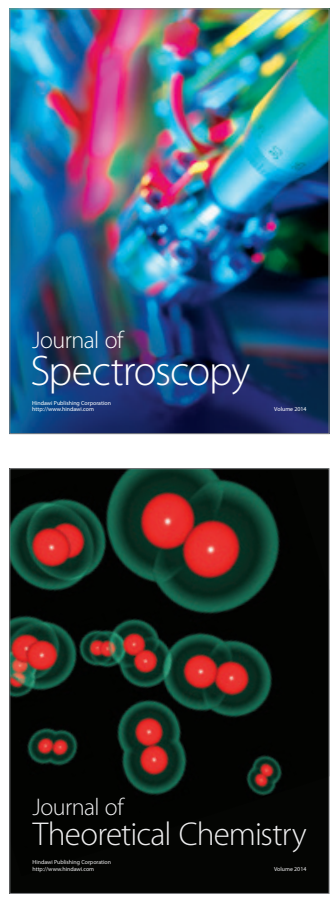
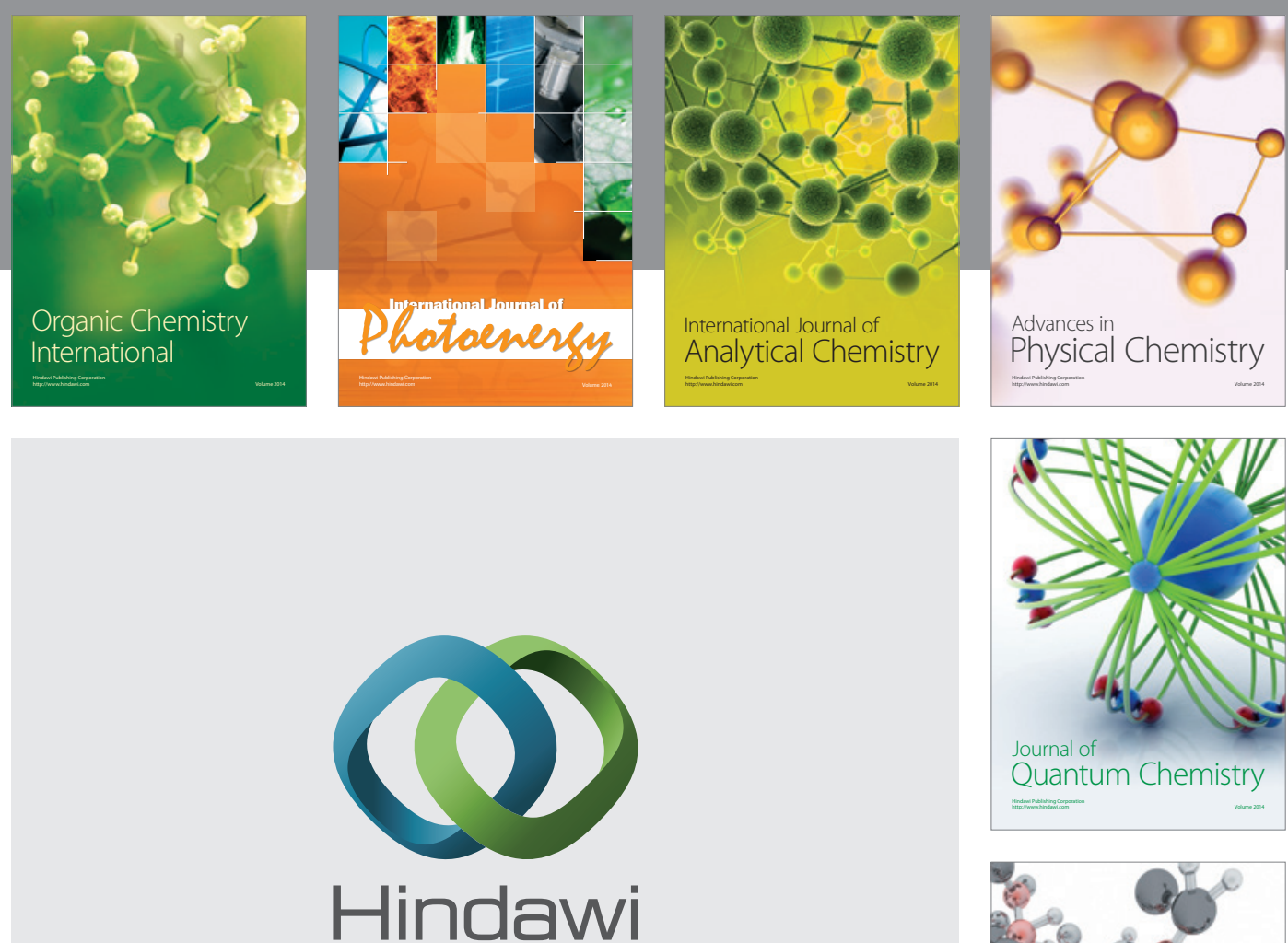

Submit your manuscripts at

http://www.hindawi.com

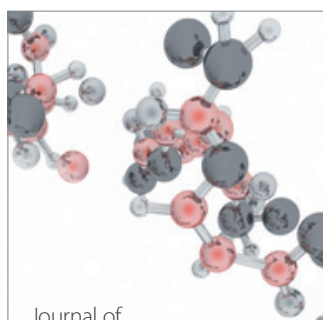

Analytical Methods

in Chemistry

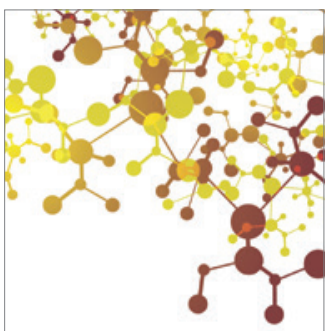

Journal of

Applied Chemistry

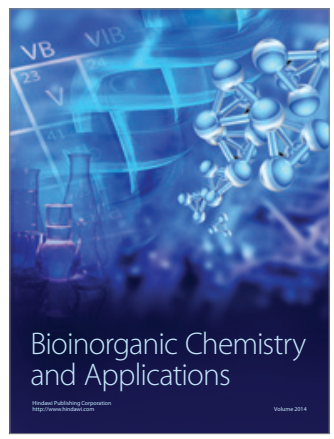

Inorganic Chemistry
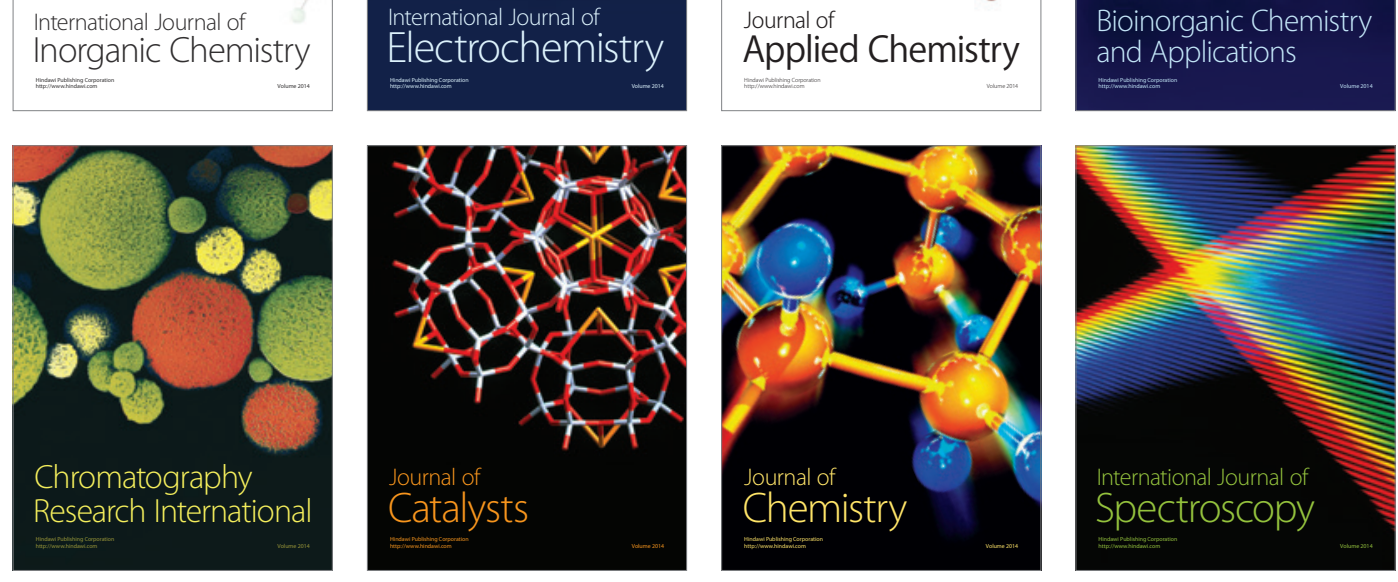